\title{
AÇÕES E ATIVIDADES DESENVOLVIDAS PELA ENFERMAGEM NO CENTRO DE ATENÇÃO PSICOSSOCIAL: REVISÃO INTEGRATIVA
}

\author{
ACTIONS AND ACTIVITIES DEVELOPED BY NURSING AT THE \\ PSYCHOSOCIAL CARE CENTER: INTEGRATIVE LITERATURE REVIEW
}

\section{ACCIONES Y ACTIVIDADES DESARROLLADAS POR LA ENFERMERÍA EN EL CENTRO DE ATENCIÓN PSICOSOCIAL: REVISIÓN INTEGRADORA}

\author{
John Victor dos Santos Silva ${ }^{1}$, Thyara Maia Brandão ${ }^{2}$, Keila Cristina Pereira do Nascimento Oliveira ${ }^{3}$
}

\begin{abstract}
RESUMO
Objetivo: descrever o que se tem produzido cientificamente sobre as ações e atividades desenvolvidas pelos profissionais de enfermagem nos Centros De Atenção Psicossocial. Metodologia: Trata-se de uma revisão integrativa da literatura, realizada nas bases de dados SciELO, LILACS e BDENF, nos meses de abril e maio, onde a amostra foi de 11 estudos. Resultados: Algumas das ações e atividades são: acolhimento, visitas domiciliares, oficinas e grupos terapêuticos, consulta familiar, organização do CAPS, reunião de equipe, atividades externas, geração de renda, PTS; além de: consulta de enfermagem, administração de medicamentos, educação em saúde, comunicação terapêutica, verificação de sinais vitais e curativos. Conclusão: As ações e atividades são bastante diversificadas, a depender do público de usuários que frequentam os CAPS, e que, na maioria, são exercidas pela equipe de enfermagem ou de forma coletiva com os demais profissionais de outras categorias. Apresentam-se, também, algumas características positivas e negativas que são fatores que podem até potencializar ou fragilizar o trabalho nos serviços.
\end{abstract}

Descritores: Enfermagem; Saúde Mental; Serviços de Saúde Mental.

\begin{abstract}
Objective: to describe what has been produced scientifically about the actions and activities developed by the nursing professionals in the Psychosocial Care Centers Methodology: It is an integrative review of the literature, carried out in the databases SciELO, LILACS and BDENF, in the months of April and May, where the sample was of 11 studies. Results: Some of the actions and activities are: reception, home visits, workshops and therapeutic groups, family consultation, CAPS organization, team meeting, external activities, income generation, PTS; in addition to: nursing consultation, medication administration, health education, therapeutic communication, verification of vital and curative signs. Conclusion: The actions and activities are quite diverse, depending on the public of users who attend the CAPS, and who, in the majority, are exercised by the nursing team or in a collective way with other professionals of other categories. Some positive and negative characteristics are also presented, which are factors that may even potentialize or weaken work in services.
\end{abstract}

Keywords: Nursing; Mental Health; Mental Health Services.

\footnotetext{
${ }_{1}^{1}$ Acadêmico de Enfermagem pela Universidade Estadual de Ciências da Saúde de Alagoas. Presidente da Liga Acadêmica Interdisciplinar de Saúde Mental (LAISME-UNCISAL).

${ }^{2}$ Enfermeira. Mestre em Enfermagem. Especialista em Psiquiatria e Saúde Mental. Professora do curso de Enfermagem da Universidade Estadual de Ciências da Saúde de Alagoas.

${ }^{3}$ Enfermeira. Doutora em Serviço Social. Mestre em Enfermagem. Professora do curso de Enfermagem da Universidade Estadual de Ciências da Saúde de Alagoas e da Universidade Federal de Alagoas.
} 


\section{RESUMEN}

Objetivo: describir lo que se ha producido científicamente sobre las acciones y actividades desarrolladas por los profesionales de enfermería en los Centros de Atención Psicosocial. Metodología: Se trata de una revisión integrativa de la literatura, realizada en las bases de datos SciELO, LILACS y BDENF, en los meses de abril y mayo, donde la muestra fue de 11 estudios. Resultados: Algunas de las acciones y actividades son: acogida, visitas domiciliarias, talleres y grupos terapéuticos, consulta familiar, organización del CAPS, reunión de equipo, actividades externas, generación de ingresos, PTS; además de: consulta de enfermería, administración de medicamentos, educación en salud, comunicación terapéutica, verificación de signos vitales y curativos. Conclusión: Las acciones y actividades bastante diversificadas, a depender del público de usuarios que frecuentan los CAPS, y que, en su mayoría, son ejercidas por el equipo de enfermería o de forma colectiva con los demás profesionales de otras categorías. Se presentan también algunas características positivas y negativas que son factores que pueden incluso potenciar o debilitar el trabajo en los servicios.

Descriptores: Enfermería; Salud Mental; Servicios de Salud Mental.

\section{INTRODUÇÃO}

A Enfermagem está presente na assistência às pessoas em sofrimento mental antes mesmo do movimento da reforma psiquiátrica, sendo suas atividades exercidas inicialmente nos hospitais psiquiátricos, onde as principais atribuições eram a vigilância, manter a ordem, o controle dos indivíduos através de medidas violentas e coercivas, além da manutenção das terapias medicamentosas e auxilio ao médico psiquiátrico nos procedimentos realizados. Essas funções caracterizavam o modelo de assistência prestado na época, centrado no controle asilar. $^{1}$

Com o advento da Reforma Psiquiátrica no final dos anos 70, iniciado com o Movimento dos Trabalhadores da Saúde Mental (MTSM), e que contavam com a participação de profissionais da enfermagem que atuavam nos hospitais psiquiátricos, começou a discussão na sociedade sobre a mudança do tratamento e assistência em saúde mental, questionando o modelo asilar vigente e bastante fomentado e financiado pelo governo e instituições privadas na época. ${ }^{1}$

Diante de todas essas mudanças de cunho social sobre a saúde mental, começaram a surgir novos serviços com características comunitárias, onde os sujeitos pudessem ser assistidos dentro da sociedade, na comunidade e não mais isolado nos hospitais. E com a aprovação da lei 10.216 em 2001, que redireciona o cuidado à pessoa com transtorno mental, essa ideia tomou maior força e respaldo nacional, legitimando as mudanças no campo da saúde mental. Foi a partir de 2002 que foram criados os Centros de Atenção Psicossocial (CAPS), que são os principais serviços de saúde mental no âmbito do Sistema Único de Saúde (SUS) 
de caráter territorial, aberto e comunitário para prestar assistência às pessoas com transtornos mentais. ${ }^{2}$

$\mathrm{Na}$ organização e estabelecimento dos CAPS, regulamentados pela publicação da portaria no 336 do Ministério da Saúde, podemos observar que o profissional de enfermagem também faz parte dos recursos humanos para prestar assistência nesse novo modelo de cuidado e atenção, dentro das modalidades e especificidades dos CAPS, como o CAPS I, II ou III, direcionados para assistência de jovens e adultos; CAPSad, para pessoas que necessitam de cuidados em saúde mental pelo uso abusivo de álcool e outras drogas; e o CAPSi, direcionado para a assistência de crianças e adolescentes com transtornos mentais. ${ }^{3}$

Após anos de conquistas e mudanças no âmbito da assistência em saúde mental, com a publicação da portaria $\mathrm{n}^{\circ} 3088$ do Ministério da Saúde, foi instituída a Rede de Atenção Psicossocial que foi criada com o intuito de articular e ampliar os pontos de atenção à saúde mental das pessoas que possuem transtornos mentais dentro do SUS, o que garantiu a integridade do cuidado para esses indivíduos, através de diversificados serviços, além dos CAPS, como as Residências Terapêuticas, leitos de saúde mental em Hospitais Gerais, Centros de
Convivência, Unidades de Recolhimento, entre outros. $^{4}$

Embora o surgimento de diversificados serviços de saúde mental, o CAPS ainda permanece como a principal estratégia utilizada por muitos municípios na assistência às pessoas com transtornos mentais. Os profissionais de enfermagem, de nível médio e superior, compõe a equipe do CAPS justamente profissionais formações e que são responsáveis por prestar os cuidados e assistência necessários à reabilitação psicossocial desses indivíduos através de diversas estratégicas terapêuticas. $^{5}$

Sabendo que a enfermagem tem papel importante nos cuidados junto à pessoa com transtornos mentais, precisamos conhecer as ações e atividades mais desenvolvidas nos serviços públicos de saúde mental, de forma a compreender como é organizado o processo de reabilitação e quais potencialidades ou vulnerabilidade o desempenho dessas ações pode causar no trabalho e na assistência dos usuários dos CAPS. ${ }^{6}$

Desta forma, o presente trabalho tem como objetivo descrever o que se tem produzido cientificamente sobre as ações e atividades desenvolvidas pelos profissionais de enfermagem nos Centros De Atenção Psicossocial. Para alcançar esse objetivo, foram estabelecidas as 
estratégias de trabalho apresentadas a seguir.

\section{MÉTODOS}

Trata-se de uma revisão integrativa da literatura, realizada a partir do copilado de resultados retirados de estudos produzidos. Essa metodologia proporciona a criação de um trabalho contendo o apanhado da produção científica sobre determinado assunto, sendo, essa técnica um instrumento da pesquisa a partir da Prática Baseada em Evidências. ${ }^{7}$

O presente trabalho foi elaborado das seguintes etapas: 1) elaboração da pergunta de pesquisa; 2) determinação da amostragem (critérios de inclusão e exclusão); 3) coleta dos dados; 4) análise dos dados; 5) discussão dos dados produzidos; e 6) apresentação da revisão. ${ }^{7}$

A pergunta que norteou $\mathrm{O}$ desenvolvimento da pesquisa foi: "O que se têm produzido cientificamente sobre as ações e atividades desenvolvidas pelos profissionais de enfermagem que trabalham nos Centros de Atenção Psicossocial?". A busca pelos artigos selecionados foi realizada nos meses de Abril e Maio de 2018.

Foi realizado uma busca nas bases de dados Scientific Electronic Library Online (SciELO), Literatura LatinoAmericana e do Caribe em Ciências da Saúde (LILACS) e no Banco de Dados em Enfermagem (BDENF), separadamente, através da seguinte estratégia: "Enfermagem” AND “Centro de Atenção psicossocial". Foram encontrados ao total 604 que foram submetidos aos critérios de inclusão: artigos publicados nos últimos cinco anos, arquivo completo disponível, nos idiomas português, inglês e espanhol. Restaram 179 artigos que foram analisados através da leitura do título e resumo. Os critérios de exclusão foram: trabalhos de revisão, monografias, dissertações ou teses. O processo de seleção dos artigos pode ser observado no quadro abaixo:

Quadro 1 - Descrição do processo de seleção dos artigos, Maceió, Alagoas, Brasil, 2018.

\begin{tabular}{|l|c|c|c|c|}
\hline \multicolumn{1}{|c|}{ Tratamento dos artigos } & SciELO & LILACS & BDENF & TOTAL \\
\hline Busca pelos descritores & 114 & 266 & 224 & 604 \\
\hline Aplicação dos critérios de inclusão e exclusão & 47 & 74 & 58 & 179 \\
\hline Após análise interna dos artigos & 5 & 4 & 2 & 11 \\
\hline
\end{tabular}

Fonte: Dados da pesquisa, 2018.

Após todo o processo de seleção dos artigos, os mesmo foram analisados de acordo com a pergunta da pesquisa e temática. Por se tratar de um trabalho que tem como objetivo retratar as ações e atividades desenvolvidas pela enfermagem 
nos Centros de Atenção Psicossocial, um dos dispositivos substitutivos da Rede de Atenção Psicossocial do $\mathrm{Brasil}^{8}$, não puderam ser incluídos trabalhos que retratassem a realidade de outros serviços ou de outros países.

Os autores utilizaram o instrumento validado por $\mathrm{Ursi}^{9}$ para análise interna e externa dos artigos e extração das informações referente aos seus conteúdos. A análise interna consistiu na retirada a partir dos resultados dos estudos todas as ações e atividades desenvolvidas pela enfermagem nos Centros de Atenção Psicossocial. A análise interna consistiu na retirada de informações como: título do artigo; base de dados onde foram encontrados, periódicos e ano de publicação; idiomas; Qualis; métodos, tipo de estudos e abordagens escolhidas para o seu desenvolvimento.

Após a análise interna dos estudos, as ações e atividades desenvolvidas que emergiram foram agrupadas em duas categorias: 1) atividades desenvolvidas exclusivamente pela enfermagem e 2) atividades desenvolvidas com os demais profissionais dos CAPS. Os dados são apresentados logo mais nos resultados.

\section{RESULTADOS}

A partir dos onze artigos selecionados para análise, foram extraídas informações referentes aos estudos. A primeira delas foi a base de dados, onde $45 \%$ estão indexados na SciELO, 36\% na LILACS e $18 \%$ na BDENF. A respeito das revistas onde os artigos foram publicados, ficou bastante diversificado os periódicos, havendo repetição da revista Texto \& contexto Enfermagem duas vezes e da Revista da Rede de Enfermagem do Nordeste três vezes, como pode ser observado no quadro abaixo.

Quadro 2 - Distribuição dos artigos selecionados e analisados, de acordo com título, ano, autores, revista, conclusão dos artigos. Maceió, Alagoas, Brasil, 2018.

\begin{tabular}{|c|l|l|c|c|c|}
\hline ESTUDO & \multicolumn{1}{|c|}{ AUTORES } & \multicolumn{1}{|c|}{ TÍTULO } & ANO & REVISTA & $\begin{array}{c}\text { BASE } \\
\text { DE } \\
\text { DADOS }\end{array}$ \\
\hline $\mathbf{1}$ & $\begin{array}{l}\text { Santos NS; } \\
\text { Esperidião E; } \\
\text { Bezerra, ALQ; } \\
\text { Cavalcante, } \\
\text { ACG; } \\
\text { Souza ACS; } \\
\text { Silva KKC. }\end{array}$ & $\begin{array}{l}\text { Percepção de Enfermeiros sobre } \\
\text { aspectos facilitadores e } \\
\text { dificultadores de suas práticas nos } \\
\text { serviços de saúde mental }{ }^{10}\end{array}$ & 2013 & $\begin{array}{c}\text { Revista } \\
\text { Brasileira de } \\
\text { Enfermagem }\end{array}$ & LILACS \\
\hline $\mathbf{2}$ & $\begin{array}{l}\text { Kantorski LP; } \\
\text { Guedes AC; }\end{array}$ & $\begin{array}{l}\text { Medicação pactuada como recurso } \\
\text { terapêtico no processo de trabalho }\end{array}$ & 2013 & $\begin{array}{l}\text { Texto \& } \\
\text { Contexto }\end{array}$ & SCIELO \\
\hline
\end{tabular}




\begin{tabular}{|c|c|c|c|c|c|}
\hline & $\begin{array}{l}\text { Feijó AM; } \\
\text { Hisse CN. }\end{array}$ & $\begin{array}{l}\text { de um CAPS: contribuições para a } \\
\text { enfermagem }^{11}\end{array}$ & & Enfermagem & \\
\hline 3 & $\begin{array}{l}\text { Lopes PF; } \\
\text { Garcia APRF; } \\
\text { Toledo VP. }\end{array}$ & $\begin{array}{l}\text { Processo de Enfermagem no } \\
\text { cotidiano do enfermeiro nos } \\
\text { Centros de Atenção Psicossocial } \\
\text { 12 }\end{array}$ & 2014 & $\begin{array}{l}\text { Revista da Rede } \\
\text { de Enfermagem } \\
\text { do Nordeste }\end{array}$ & BDENF \\
\hline 4 & $\begin{array}{l}\text { Almeida ANS; } \\
\text { Feitosa RMM; } \\
\text { Boesmans EF; } \\
\text { Silveira LC. }\end{array}$ & $\begin{array}{l}\text { Cuidado Clínico de Enfermagem } \\
\text { em Saúde Mental: Reflexão sobre a } \\
\text { prática do Enfermeiro }^{13}\end{array}$ & 2014 & $\begin{array}{l}\text { Revista de } \\
\text { Pesquisa: } \\
\text { Cuidado é } \\
\text { Fundamental }\end{array}$ & LILACS \\
\hline 5 & $\begin{array}{l}\text { Maynart WHC; } \\
\text { Albuquerque } \\
\text { MCS; } \\
\text { Brêda MZ; } \\
\text { Jorge JS. }\end{array}$ & $\begin{array}{l}\text { A escuta qualificada e o } \\
\text { acolhimento na Atenção } \\
\text { Psicossocial }{ }^{14}\end{array}$ & 2014 & $\begin{array}{l}\text { Acta Paulista de } \\
\text { Enfermagem }\end{array}$ & SCIELO \\
\hline 6 & $\begin{array}{l}\text { Moll MF, } \\
\text { Alves JF, } \\
\text { Silva DC, } \\
\text { Faria TDS, } \\
\text { Ventura CAA, } \\
\text { Silva LD. } \\
\end{array}$ & $\begin{array}{l}\text { Ações terapêuticas para pessoas } \\
\text { com esquizofrenia acompanhadas } \\
\text { num Centro de Atenção } \\
\text { Psicossocial }^{15}\end{array}$ & 2015 & $\begin{array}{l}\text { Revista } \\
\text { Portuguesa de } \\
\text { Enfermagem de } \\
\text { Saúde Mental }\end{array}$ & SCIELO \\
\hline 7 & $\begin{array}{l}\text { Franzoi MAH; } \\
\text { Santos JLG; } \\
\text { Backes VMS; } \\
\text { Ramos FRS. }\end{array}$ & $\begin{array}{l}\text { Intervenção musical como } \\
\text { estratégia de cuidado em } \\
\text { enfermagem a crianças com } \\
\text { Transtorno do Espectro Autimo em } \\
\text { um Centro de Atenção } \\
\text { Psicossocial }^{16}\end{array}$ & 2016 & $\begin{array}{l}\text { Texto \& } \\
\text { Contexto } \\
\text { Enfermagem }\end{array}$ & SCIELO \\
\hline 8 & $\begin{array}{l}\text { Salimena AMO; } \\
\text { Ferrugini RRB; } \\
\text { Melo MCSA; } \\
\text { Amorim TV. } \\
\end{array}$ & $\begin{array}{l}\text { Compreensão da espiritualidade } \\
\text { para os portadores de transtorno } \\
\text { mental: contribuições para o } \\
\text { cuidado de enfermagem }^{17}\end{array}$ & 2016 & $\begin{array}{l}\text { Revista Gaúcha } \\
\text { de Enfermagem }\end{array}$ & SCIELO \\
\hline 9 & $\begin{array}{l}\text { Brandão TM; } \\
\text { Brêda MZ; } \\
\text { Nascimento } \\
\text { YCML; } \\
\text { Albuquerque } \\
\text { MCS; } \\
\text { Albuquerque RS. }\end{array}$ & $\begin{array}{l}\text { A práxis do enfermeiro na atenção } \\
\text { psicossocial: vulnerabilidades e } \\
\text { potencialidades presentes }^{18}\end{array}$ & 2016 & $\begin{array}{l}\text { Revista de } \\
\text { Enfermagem da } \\
\text { UFPE }\end{array}$ & BDENF \\
\hline 10 & $\begin{array}{l}\text { Biffi D; } \\
\text { Nasi C. }\end{array}$ & $\begin{array}{l}\text { Expectativas de usuários sobre a } \\
\text { prática de enfermeiros de um } \\
\text { Centro de Atenção } \\
\text { Psicossocial }^{19}\end{array}$ & 2016 & $\begin{array}{l}\text { Revista da Rede } \\
\text { de Enfermagem } \\
\text { do Nordeste }\end{array}$ & LILACS \\
\hline 11 & $\begin{array}{l}\text { Fortes FLS, } \\
\text { Peres MAA, } \\
\text { Santos TCF, } \\
\text { Martins GCS, } \\
\text { Montenegro } \\
\text { HRA, } \\
\text { Almeida Filho } \\
\text { AJ. }\end{array}$ & $\begin{array}{l}\text { Enfermeiro em saúde mental: } \\
\text { concepções sobre qualificação } \\
\text { profissional em um Centro de } \\
\text { Atenção Psicossocial }{ }^{20}\end{array}$ & 2017 & $\begin{array}{l}\text { Revista da Rede } \\
\text { de Enfermagem } \\
\text { do Nordeste }\end{array}$ & LILACS \\
\hline
\end{tabular}

Fonte: Dados da pesquisa, 2018

$\mathrm{O}$ ano de publicação dos artigos também foi bastante variado, onde pôde-se constatar que $36 \%$ dos artigos foram publicados no ano de 2016, 27\% em 2014,
$18 \%$ em 2013, $9 \%$ em 2015, e $9 \%$ em 2017. Todos os artigos foram publicados no Brasil, levando em consideração o objeto de estudo relacionado às 
características da enfermagem nos Centros De Atenção Psicossocial, um serviço do Brasil $^{8}$, tornando inviável a inclusão de artigos que retratassem a realidade dos serviços de atenção em saúde mental de outros países. Referente ao idioma de publicação, $55 \%$ foi publicado em português e inglês, $36 \%$ apenas em português e $9 \%$ apenas em inglês.

Foi possível também verificar o Qualis para a área de Enfermagem das revistas onde os artigos foram publicados, sendo 45\% com Qualis B1, 36\% com Qualis A2 e 18\% com Qualis B2. Isso pode demonstrar que os estudos seguem um rigor metodológico e com certa qualidade nas publicações, tendo em vista a concentração das publicações em revista de alto Qualis.

A respeito dos métodos empregados nos artigos, foi analisado que $100 \%$ das pesquisas utilizaram a abordagem qualitativa, e apenas $82 \%$ desses deixaram claro o método adotado. Sobre o tipo de estudo, observou-se que $73 \%$ dos artigos deixaram o tipo de estudo claro, onde a maioria utilizou o estudo descritivo e o exploratório, associado a outros tipos de estudos, como o fenomenológico, histórico-social e relato de experiência.

Quanto ao conteúdo dos artigos, $74 \%$ dos artigos analisados tratavam-se das práticas e o processo de trabalho da enfermagem nos serviços de saúde mental e os outros $26 \%$ tratavam-se das condições de trabalho, formação dos profissionais e o impacto na vida de no trabalho dos profissionais de enfermagem. Desta forma, as ações e atividades desenvolvidas pelos profissionais de enfermagem encontrados nos estudos estão descritas no quadro 3. Algumas dessas atividades são compartilhadas com os demais profissionais, outras são de exclusividade dos profissionais de enfermagem.

Quadro 3 - Ações e Atividades desenvolvidas pela Enfermagem nos CAPS, Maceió, Alagoas, Brasil, 2018.

\begin{tabular}{|c|c|}
\hline Enfermagem e demais categorias profissionais & Equipe de Enfermagem \\
\hline Acolhimento $^{5,8}$ & Supervisão da Equipe de Enfermagem ${ }^{1,11}$ \\
\hline Visitas Domiciliares $^{3,4,10}$ & Sistematização da Assistência de Enfermagem ${ }^{1,11}$ \\
\hline Oficinas e Grupos Terapêuticos ${ }^{6,7}$ & Consulta de Enfermagem ${ }^{5,10}$ \\
\hline Consulta Familiar ${ }^{4,3}$ & Administração de Medicamentos ${ }^{2,3}$ \\
\hline Organização do CAPS ${ }^{1,3}$ & Educação em Saúde ${ }^{4,9}$ \\
\hline Reunião de Equipe ${ }^{1,11}$ & Comunicação Terapêutica ${ }^{5,8}$ \\
\hline Atividades externas com os Usuários ${ }^{1,9}$ & Verificação dos Sinais Vitais ${ }^{3,9}$ \\
\hline Projeto Terapêutico Singular 8,9 & Realização de Pequenos Curativos ${ }^{3,9}$ \\
\hline
\end{tabular}

Fonte: Dados da pesquisa, 2018. 
Além dessas ações e atividades desenvolvidas, foi possível também encontrar algumas características positivas e negativas sobre a enfermagem nos estudos e que podem ser fatores de potencialidades ou vulnerabilidades do trabalho desses profissionais nos CAPS, como mostra o quadro abaixo:

Quadro 4 - Características Positivas e Negativas da Enfermagem nos CAPS, Maceió, Alagoas, Brasil, 2018.

\begin{tabular}{|c|c|}
\hline Características Positivas & Características Negativas \\
\hline $\begin{array}{l}\text { Profissionais que mais criam vínculos com os usuários } \\
\text { dos serviços } 12,17,19,20\end{array}$ & $\begin{array}{l}\text { Falta de preparo dos Profissionais da Enfermagem } \\
\qquad 15,19,20\end{array}$ \\
\hline Facilidade no trabalho multiprofissional ${ }^{13,17}$ & Sobrecarga no Trabalho ${ }^{10,16,18}$ \\
\hline $\begin{array}{l}\text { Participa ativamente do processo de reabilitação } \\
\text { Psicossocial }^{12,14}\end{array}$ & $\begin{array}{c}\text { Trabalham com pouco recurso e em estruturas } \\
\text { desfavoráveis }{ }^{10,16}\end{array}$ \\
\hline
\end{tabular}

Fonte: Dados da pesquisa, 2018

\section{DISCUSSÃO}

O profissional de enfermagem, além de uma exigência da política de saúde mental, é um profissional necessário para desempenhar atividades relacionadas à assistência à pessoa em sofrimento mental. O profissional de enfermagem, seja ele de nível superior ou médio, contribui de forma significativa para o trabalho nos CAPS. $^{6}$

Assim, entre algumas das atribuições específicas da enfermagem estão: utilização da Sistematização da Assistência de Enfermagem (SAE) e o Processo de Trabalho da Enfermagem (PTE) que direciona e organiza o cuidado de Enfermagem para os indivíduos e familiares. Ações relacionadas ao fazer da enfermagem, como administração de medicamentos, verificação de sinais vitais, realização de consulta de enfermagem aos usuários e familiares. Os profissionais de enfermagem acabam por ser responsáveis, muitas vezes, pelas medicações dos usuários. Eles orientam sobre o uso da medicação, sobre os efeitos colaterais, a importância da terapêutica medicamentosa, algumas vezes administrando no próprio CAPS os medicamentos. ${ }^{21}$

Os profissionais de enfermagem participam constantemente das ações de educação em saúde nos CAPS. Trabalhar a promoção da saúde e prevenção dos agravos é algo intrínseco do fazer da enfermagem, pois está diretamente liga a sua formação para a qualidade de vida e assistência em saúde dos sujeitos Desta forma, quando necessário, a equipe de enfermagem realiza a verificação dos sinais vitais dos usuários, além de realizar procedimentos como pequenos curativos. 
Esses procedimentos dependem dos materiais disponíveis nos serviços. ${ }^{22}$

Outra atividade muito desempenhada pela enfermagem nos CAPS são as visitas Domiciliares, que é onde esses profissionais realizam a busca ativa pelos usuários e seus familiares quando os mesmos desistem do tratamento, prezando pela sua. Isso é uma preocupação constante desses profissionais. As visitas domiciliares, algumas vezes, são comprometidas pela falta de transporte, mas os profissionais fazem o possível para manter a assistência sem prejuízos. ${ }^{6}$

Os profissionais de enfermagem também realizam atividades externas com os usuários, tudo isso está dentro dos Projetos Terapêuticos Singulares, que são construídos entre profissionais e usuários, de forma a priorizar a reabilitação psicossocial desses sujeitos favorecendo a autonomia. A enfermagem participa ativamente dessa construção, através do enfermeiro, que é quem da equipe tem formação para esse trabalho. ${ }^{6}$

Uma característica bastante interessante dos profissionais de enfermagem é que eles acabam sendo os profissionais que os usuários mais criam vínculo dentro do serviço. A enfermagem promove o acolhimento dentro do serviço, o que não signifique que seja uma exclusividade desses profissionais, porém identificamos que eles são os que mais mantêm contato com os usuários. Essa característica da enfermagem estar sempre presente é inerente à profissão, visto que na maioria dos serviços de saúde os profissionais de enfermagem são os que estão em maior contato com os usuários, o que possibilita a criação de vínculo e confiança. Isso também vai depender do profissional estar aberto para tal acontecimento. $^{22}$

Sobre as práticas de enfermagem na assistência através da reabilitação psicossocial junto à pessoa em sofrimento mental, podemos identificar o uso da comunicação terapêutica, a escuta qualificada, participação na criação do Projeto Terapêutico Singular, participação das oficinas terapêuticas, participação nas atividades de geração de renda, entre outras. A enfermagem pode contribuir no processo de reabilitação psicossocial do usuário, como qualquer profissional do serviço, embora muitas vezes essas atividades fiquem limitadas apenas aos psicólogos, terapeutas ocupacionais, oficineiros, entre outros. ${ }^{6}$

A enfermagem também desempenha suas atividades de forma interdisciplinar, com a participação de outros profissionais, da mesma forma que ela contribui participando das atividades dos demais profissionais. Verificou-se que 
a enfermagem não tem problemas em desempenhas suas atividades em grupo, com os demais profissionais, o que fortalece $o$ processo de reabilitação psicossocial, quando o trabalho é exercido de forma conjunta, e não apenas individual. $\mathrm{O}$ trabalho em equipe nos CAPS é primordial e depende da perspectiva do profissional sobre o seu papel e o papel dos demais da equipe. A forma como o profissional enxerga a equipe pode interferir diretamente nas atividades do serviço. ${ }^{5}$

Uma preocupante questão identificada nos estudos é a pouca formação e capacitação da enfermagem para o trabalho nos serviços de saúde mental. Poucos chegam a fazer curso de especialização ou capacitação. A formação em saúde mental para os profissionais de enfermagem é uma questão que permeia a graduação, mas muitas vezes os conteúdos são fragmentados e superficiais, limitados, o que pode não preparar adequadamente o profissional para trabalhar na assistência à pessoa em sofrimento mental ${ }^{23}$. Foi percebido também que a maioria dos profissionais não recebe capacitação para o trabalho por parte dos órgãos que gerenciam o serviço, o que pode impactar diretamente na assistência direta ao usuário do serviço, uma vez que esses profissionais podem não compreender qual o seu papel na assistência. Desta forma, eles acabam por aprender a trabalhar na prática no cotidiano do CAPS de acordo com as demandas. $^{24}$

Percebe-se, também, que a enfermagem muitas vezes trabalha em serviços onde a estrutura é precária e com poucos recursos e materiais para desenvolver as atividades terapêuticas, o que pode provocar o desgaste físico e psicológico dos profissionais, pelo esforço exercido para prestar uma assistência completa, mesmo em situações dificuldades, o que é um retrato da maioria dos serviços de saúde mental no Brasil ${ }^{25}$.

\section{CONCLUSÃO}

O estudo mostra que a enfermagem que atua nos Centros de Atenção Psicossocial desempenha ações e atividades bastante diversificadas, a depender do público de usuários que frequentam os CAPS, e que, na maioria, são exercidas pela equipe de enfermagem ou de forma coletiva com os demais profissionais de outras categorias. Apresentam-se, também, algumas características positivas e negativas que são fatores que podem até potencializar ou fragilizar o trabalho nos serviços.

Percebe-se que as atividades desempenhadas pela enfermagem estão mais relacionadas ao exercício próprio da 
profissão e que são importantes para o funcionamento dos CAPS. Em sua maioria, os estudos demonstram que esses profissionais possuem uma parcela significativa para a efetivação da assistência nestes serviços, atuando diretamente com os usuários dos CAPS.

$$
\text { Embora as atividades }
$$

desempenhadas pela enfermagem estejam mais relacionadas ao processo de trabalho da profissão, o profissional de enfermagem pode contribui significativamente no processo de reabilitação psicossocial de forma coletiva.

A limitação deste trabalho está no número pequeno de artigos estudados, o que pode não retratar completamente as ações e atividades desenvolvidas por esses profissionais nos serviços do Brasil. Sugerimos que sejam realizadas novas pesquisas para conhecer o processo de trabalho da enfermagem que trabalha e atua nos Centros de Atenção Psicossocial.

\section{REFERÊNCIAS}

1. Esperidião E, Silva NS, Caixeta CC, Rodrigues J. A Enfermagem Psiquiátrica, a ABEn e o Departamento Científico de Enfermagem Psiquiátrica e Saúde Mental: avanços e desafios. Rev Bras Enferm. 2013; 66(esp.):171-76.

2. Costa JP, Jorge MSB, Coutinho MPL, Costa EC, Holanda ITA. A reforma psiquiátrica e seus desdobramentos: representações sociais dos profissionais e usuários da atenção psicossocial. Psicol Saber Soc. 2016; 5(1):35-45.
3. Fernandes RL, Miranda FAN. Análise da teoria das relações interpessoais: cuidado de enfermagem nos centros de atenção psicossocial. Rev Enferm UFPE on line [Internet]. 2016 [citado em $22 \mathrm{abr}$ 2018]; 10(2):880-86. Disponível em: https://periodicos.ufpe.br/revistas/revistaen fermagem/article/view/11032/12425 4. Ministério da Saúde (Brasil). Portaria ${ }^{\circ}$ 3.088, de 23 de dezembro de 2011. Institui a Rede de Atenção Psicossocial para pessoas com sofrimento ou transtorno mental e com necessidades decorrentes do uso de crack, álcool e outras drogas, no âmbito do Sistema Único de Saúde [Internet]. Brasília, DF: Ministério da Saúde; 2011 [citado em 22 abr 2018]. Disponível em:

http://bvsms.saude.gov.br/bvs/saudelegis/g m/2011/prt3088_23_12_2011_rep.html.

5. Vasconcelos MGF, Jorge MSB, Catrib AMF, Bezerra IC, Franco TB. Projeto terapêutico em Saúde Mental: práticas e processos nas dimensões constituintes da atenção psicossocial. Interface (Botucatu) [Internet]. 2016 [citado em 22 abr 2018]; 20(57):313-23. Disponível em:

http://www.scielo.br/pdf/icse/v20n57/1807 -5762-icse-20-57-0313.pdf

6. Dias CB, Silva ALA. O perfil e a ação profissional da(o) enfermeira(o) no Centro de Atenção Psicossocial. Rev Esc Enferm USP. 2010; 44(2):469-75.

7. Souza MT, Silva MD, Carvalho R. Revisão integrativa: o que é e como fazer. Einstein (São Paulo). 2010; 8(1):102-6.

8. Quinderé PHD, Jorge MSB, Franco TB. Rede de atenção psicossocial: qual o lugar da saúde mental? Physis. 2014; 24(1):25371.

9. Ursi ES, Galvão CM. Prevenção de lesões de pele no perioperatório: revisão integrativa da literatura. Rev Latinoam Enferm. 2006; 14(1):124-31. 10. Silva NS, Esperidião E, Bezerra ALQ, Cavalcante ACG, Souza ACS, Silva KKC. Percepção de enfermeiros sobre aspectos facilitadores e dificultadores de sua prática 
nos serviços de saúde mental. Rev Bras Enferm. 2013; 66(5):745-52

11. Kantorski LP, Guedes AC, Feijó AM, Hisse CN. Medicação pactuada como recurso terapêutico no processo de trabalho de um CAPS: contribuições para a enfermagem. Texto \& Contexto Enferm. 2013; 22(4):1022-29.

12. Lopes PF, Garcia APRF, Toledo VP. Processo de Enfermagem no cotidiano do enfermeiro nos Centros de Atenção Psicossocial. Rev Rene. 2014; 15(5):780-8. 13. Almeida ANS, Feitosa RMM, Boesmans EF, Silveira LC. Cuidado clínico de enfermagem em saúde mental: reflexões sobre a prática do enfermeiro. Rev Pesqui Cuid Fundam. [Internet]. 2014 [citado em 22 abr 2018]; 6(1):213-31. Disponível em: http://www.redalyc.org/articulo.oa?id=505 750621016

14. Maynart WHC, Albuquerque MCS, Brêda MZ, Jorge JS. A escuta qualificada eo acolhimento na atenção psicossocial. Acta Paul Enferm. 2014; 27(4):300-4. 15. Moll MF, Alves JF, Silva DC, Faria TDS, Ventura CAA, Silva LD. Ações terapêuticas para pessoas com esquizofrenia acompanhadas num Centro de Atenção Psicossocial. Rev Port Enferm Saúde Mental. 2015; (14):24-30.

16. Franzoi MAH, Santos JLG, Backes VMS, Ramos FRS. Intervenção musical como estratégia de cuidado de enfermagem a crianças com Transtorno do Espectro do Autismo em um centro de atenção psicossocial. Texto \& Contexto Enferm. [Internet]. 2016 [citado em 22 abr 2018]; 25(1):e1020015. Disponível em: http://www.scielo.br/pdf/tce/v25n1/01040707-tce-25-01-1020015.pdf 17. Salimena AMO, Ferrugini RRB, Melo MCSC, Amorim TV. Compreensão da espiritualidade para os portadores de transtorno mental: contribuições para o cuidado de enfermagem. Rev Gaúch Enferm. [Internet]. 2016 [citado em $22 \mathrm{abr}$ 2018]; 37(3):e51934. Disponível em: http://www.scielo.br/pdf/rgenf/v37n3/0102 -6933-rgenf-1983-144720160351934.pdf 18. Brandão TM, Nascimento YCML, Brêda MZ, Albuquerque MCS, Albuquerque RS. A práxis do enfermeiro na atenção psicossocial: vulnerabilidades e potencialidades presentes. Rev Enferm UFPE on line. [Internet]. 2016 [citado em 22 abr 2018]; 10(6):4766-77. Disponível em:

https://periodicos.ufpe.br/revistas/revistaen fermagem/article/view/11255/12875 19. Biffi D, Nasi C. Expectativas de usuários sobre a prática de enfermeiros de um Centro de Atenção Psicossocial. Rev Rene. 2016; 17(6):789-96.

20. Fortes FLS, Peres MAA, Santos TCF, Martins GCS, Montenegro HRA, Almeida Filho AJ. Enfermeiro em saúde mental: concepções sobre qualificação profissional em um Centro de Atenção

Psicossocial. Rev Rene. 2017; 18(6):76370.

21. Cardoso LS, Santos DF, Ribeiro JP, Silva PA, Mota MS. Assistência de enfermagem desenvolvida em um centro de atenção psicossocial. Vittalle. 2015; 27(1):41-49.

22. Machado MH, Wermelinger M, Vieira M, Oliveira E, Lemos W, Aguiar Filho W, et al. Aspectos gerais da formação da enfermagem: o perfil da formação dos enfermeiros técnicos e auxiliares. Enferm Foco. 2016; 7(esp.):15-34.

23. Rosa RPF, Andrade ALF, Oliveira SP, Silva AGL, Ferreira AM, Inácio JS, et al. Construindo saberes no trabalho em saúde mental: experiências de formação em saúde. Interface Comun Saúde Educ. 2015; 19(suppl1):931-940.

24. Moura GA, Roncalli AG, Noro LRA. Impacto do trabalho em profissionais de serviços de saúde mental em um município do nordeste brasileiro. Psicol Ciênc Prof. 2016; 36(2):401-10.

25. Alves SR, Santos RP, Oliveira RG, Yamaguchi MU. Serviços de saúde mental: percepção da enfermagem em relação à sobrecarga e condições de trabalho. Rev 
Pesqui Cuid Fundam. [Internet] 2018

jan/mar [citado em 22 abr 2018]; 10(1):2529. Disponível em:

http://www.seer.unirio.br/index.php/cuidad ofundamental/article/view/5929/pdf_1

RECEBIDO: 07/08/2018

APROVADO: $12 / 11 / 2018$

PUBLICADO: $12 / 2018$ 